\title{
Electron Transfer Properties of Alternant Hydrocarbons in Terms of Inverse Adjacency Matrices of Molecular Graphs
}

\author{
Viktorija Gineityte \\ Institute of Theoretical Physics and Astronomy, Vilnius University, Gostauto 12, LT-01108 Vilnius, Lithuania \\ (E-mail:Viktorija.Gineityte@tfai.vu.lt) \\ RECEIVED APRIL 8, 2013; REVISED MAY 7, 2014; ACCEPTED JULY 15, 2014
}

\begin{abstract}
The study addresses electron transfer (ET) properties of systems of the type D-AH-A, where D and $\mathrm{A}$ are external electron-donating and accepting subsystems, respectively, and $\mathrm{AH}$ is an alternant hydrocarbon playing the role of the bridge. The main attention is paid to the dependence of the ET properties upon the sites of the $\mathrm{AH}$, the external subsystems are attached to. As is usual in one-electron models of ET properties, the latter are considered to be proportional to off-diagonal Hamiltonian matrix elements (resonance parameters) representing effective interactions of orbitals of external groups $\mathrm{D}$ and A via the bridge. The block-diagonalization procedure for the common Hückel type Hamiltonian matrix of AHs is applied to derive explicit algebraic expressions for the above-specified decisive parameters. As a result, a relation is established between these parameters and elements of the inverse adjacency matrix (IAM) of the graph of the given AH referring to sites (vertices) under consideration. The common constitution of IAMs of all AHs then yields a simple and general selection rule for the effective parameters concerned. Moreover, the unified IAM of AHs is shown to be expressible in terms of entire submatrices (blocks) of the initial AM. This allows the ET properties of composite AHs to be analyzed in terms of increments of separate fragments (subgraphs) and of their interaction. The results of the study are illustrated by consideration of specific examples including the simplest benzenoids.
\end{abstract}

Keywords: electron transfer, alternant hydrocarbons, inverse adjacency matrix, molecular graph, blockdiagonalization transformation

\section{INTRODUCTION}

Single electron transfer forms the basis of numerous fundamental chemical and biochemical processes, therefore it is studied intensively. ${ }^{1-5}$ An additional interest in this phenomenon arises in connection with its potential applications in nanoscale science and technology. ${ }^{6-8}$ This especially refers to the so-called bridge-assisted electron transfer, where the system concerned contains an electron-donating subsystem (D) and an electronaccepting one (A) joined with a bridge (or several bridges), the latter usually coinciding with an extended organic molecule (molecules). It is evident that the electron transfer (ET) properties of the bridge(s) become of great importance in this case.

So far as constitutions of bridges themselves are concerned, a certain shift in emphasis took place during the last several decades from saturated (sigma-bounded) bridges ${ }^{4,9}$ to conjugated and/or aromatic ones. ${ }^{10-20} \mathrm{An}$ important point in this context is that the ET properties of an aromatic bridge are expected to depend upon the type of its connection to the external subsystems, i.e. a site-dependent electron transfer is predicted in this case. ${ }^{10,20}$ For systems wherein aromatic hydrocarbons (such as naphthalene, phenanthrene and anthracene) play the role of bridges, the structure-ET property relationships have been successfully interpreted recently in terms of phases and amplitudes of the relevant frontier molecular orbitals (HOMOs and LUMOs). ${ }^{10}$

In the framework of the simplest one-electron model ( $c f$. the Hückel (HMO) theory ${ }^{21,22}$ ), the majority of conjugated and/or aromatic hydrocarbons belong to the alternant systems ${ }^{21}$ representable by Hamiltonian matrices of a certain unified constitution. ${ }^{22-24}$ In this connection, numerous common properties of the relevant electronic structures have been established ${ }^{21-33}$ that are considered nowadays as classical results of quantum chemistry. Recently, these achievements have been supplemented with rules embracing various derivatives of alternant hydrocarbons (AHs). ${ }^{34-37}$ Moreover, adequacy of one-electron models for investigation of the single electron transfer is a well- 
established fact. ${ }^{10,38,39}$ On the basis of the abovementioned experience, we may then also expect some common rules to govern the AH-assisted ET processes. The principal aim of the present study consists in revealing and formulating of just these rules.

The first step towards achieving the above-specified end consists in revealing the principal characteristic of the electronic structure of an $\mathrm{AH}$ that is responsible for its consequent ET properties in the systems of the type DAH-A. In this connection, we start with constructing a simple model of the above-specified systems in the framework of the Hückel type approximation in the second Section. Thereupon, we derive and analyze general algebraic expressions for Hamiltonian matrix elements representing the effective bridge-assisted interactions between subsystems D and A in the same Section. In the next Section, we turn to particular types of AHs. The final Section is devoted to illustration of the results by consideration of specific examples.

\section{THE PRINCIPAL FORMULA FOR THE EFFEC- TIVE ALTERNANT-HYDROCARBON-ASSISTED INTERACTION OF EXTERNAL GROUPS}

Let the electron-donating subsystem (D) of our system D$\mathrm{AH}-\mathrm{A}$ be represented by a single basis orbital for simplicity, e.g. by its HOMO. Analogously, a single orbital (LUMO) will be ascribed to the electron-accepting subsystem (A). Let these orbitals be correspondingly denoted by $\psi_{d}$ and $\psi_{a}$. It deserves an immediate mention that occupation numbers (populations) of these orbitals are irrelevant to analysis of efficiency of their indirect (bridge-assisted) interaction as discussed below. Finally, the intervening alternant hydrocarbon (the bridge) will be described by the respective set of $2 \mathrm{p}_{z}$ AOs of carbon atoms $\{\chi\}$ as usual. $^{21-37}$

In general, the ET properties of a certain system are representable by Hamiltonian matrix elements between wave functions of the relevant many-electron states (the so-called ET matrix elements). ${ }^{4,40-42}$ In many cases, however, these elements may be replaced by their oneelectron analogues ${ }^{43,44}$ that are alternatively called effective resonance parameters (especially if the Hückel type model is applied). These parameters refer to basis orbitals of external subsystems (D and A) instead of wave functions of many-electron states and embrace both direct (through-space) and indirect (bridge-assisted) interactions of these orbitals. The above-introduced basis functions $\psi_{d}$ and $\psi_{a}$ evidently play the role of interacting orbitals in our case. Under the common assumption about coinciding initial one-electron energies of orbitals $\psi_{d}$ and $\psi_{a}, 38,45$ the ET property of the given bridge is proportional to the actual splitting energy $(\Delta)$ of the relevant two energy levels in addition. ${ }^{43,46-49}$
The block-diagonalization transformation for matrices ${ }^{50-53}$ was shown to be applicable ${ }^{9}$ to derive oneelectron matrix elements (effective resonance parameters) for indirect interactions of external groups via saturated (sigma-bounded) bridges. Indeed, this transformation yields a new Hamiltonian matrix of the whole system in the form of a direct sum of several nonzero Hermitian blocks (the so-called eigenblocks), each of them corresponding to an individual subset of basis functions. Given that orbitals $\psi_{d}$ and $\psi_{a}$ make a separate subset, the effective resonance parameter being sought coincides with the only off-diagonal element of the respective $2 \times 2$-dimensional eigenblock. An extension of this efficient approach to bridges under present interest evidently is highly desirable.

The above-cited study ${ }^{9}$ was based on employment of a specific version of the perturbation theory (PT) when block-diagonalizing the initial Hamiltonian matrix, namely of the so-called non-commutative Rayleigh-Schrödinger PT (NCRSPT) ${ }^{51,53}$ Requirements underlying this PT consist in small relative values of intersubset resonance parameters $v s$. the respective energy gaps and were shown to be met in the case of saturated bridges. When addressing the unsaturated (alternant) bridges, however, we have to deal with subsets of basis functions of similar one-electron energies as discussed below and an essential generalization of the whole approach is required.

As already mentioned, the Hückel type Hamiltonian matrices of AHs take a common form in the basis of $2 \mathrm{p}_{z}$ AOs of carbon atoms $\{\chi\} .^{22-24}$ For this particular matrix, an algebraic (non-perturbative) solution of the relevant block-diagonalization problem has been obtained. ${ }^{32}$ Moreover, the transformation matrices concerned also proved to be uniform for all AHs. Employment of this transformation to the total Hückel type Hamiltonian matrix of our systems D-AHA makes the first step of the subsequent derivation. The resulting Hamiltonian matrix, however, is not yet completely block-diagonal in the present case in contrast to that of an isolated $\mathrm{AH}$. Thus, the second and the final step of the whole procedure consists in invoking the perturbative approach. ${ }^{50-53}$

Let us confine ourselves here to the case of even AHs and turn to constructing the initial Hamiltonian matrix. In the simple Hückel model, the $2 n$-dimensional basis set $\{\chi\}$ of such an $\mathrm{AH}$ consisting of $2 \mathrm{p}_{\mathrm{z}}$ AOs of carbon atoms is known to be divisible into two $n$ dimensional subsets $\left\{\chi^{*}\right\}$ and $\left\{\chi^{\circ}\right\}$ so that the intrasubset resonance parameters take zero values. ${ }^{22-24}$ Given that the Coulomb parameters $\left(\alpha_{C}\right)$ are additionally assumed to be uniform and the equality $\alpha_{C}=0$ is accepted for convenience, the common model Hamiltonian matrix of AHs contains non-zero submatrices (blocks) in its off-diagonal positions only. 
These submatrices will now be included into the zero order Hamiltonian matrix of our system D-AH-A $\left(\boldsymbol{H}_{(0)}\right)$ exhibited below. Let us also invoke the usual assumption $^{38,45}$ about uniform one-electron energies of external orbitals $\psi_{d}$ and $\psi_{a}$ and suppose them to be equal to our reference point $\alpha_{C}=0$ for simplicity. Finally, let the overall relative order of basis functions coincide with $\left\{\chi^{*}\right\}, \psi_{d}, \psi_{a},\left\{\chi^{\circ}\right\}$. We then obtain

$$
\boldsymbol{H}_{(\mathbf{0})}=\left|\begin{array}{cccc}
\mathbf{0} & \mathbf{0} & \mathbf{0} & \boldsymbol{B} \\
\mathbf{0} & 0 & 0 & \mathbf{0} \\
\mathbf{0} & 0 & 0 & \mathbf{0} \\
\boldsymbol{B}^{+} & \mathbf{0} & \mathbf{0} & \mathbf{0}
\end{array}\right|,
$$

where the submatrices $\boldsymbol{B}$ and $\boldsymbol{B}^{+}$contain the intersubset resonance parameters inside the $\mathrm{AH}$ [The superscript + here and below represents the Hermitian-conjugate (transposed) matrix]. Non-zero elements $\boldsymbol{B}_{i j}$ coincide with resonance parameters referring to the neighboring pairs of AOs. The mean value of these parameters $(\beta)$ will serve as a (negative) energy unit in our study, i.e. the equality $\beta=1$ will be accepted (as is usual in the standard HMO theory ${ }^{21}$ ). Under this condition, the submatrices $\boldsymbol{B}$ and $\boldsymbol{B}^{+}$coincide with those of the adjacency matrix (AM) of the graph of the C-skeleton ${ }^{22,54,55}$ of the respective $\mathrm{AH}$ as exhibited later in Eq. 20. It also deserves mention that $\boldsymbol{B}$ and $\boldsymbol{B}^{+}$are square matrices for the most common case of classical even AHs.

Let us introduce now a certain direct interaction between orbitals $\psi_{d}$ and $\psi_{a}$. The relevant resonance parameter will be denoted by $\gamma$. Interactions (resonance parameters) between orbitals $\psi_{d}$ and $\psi_{a}$, on the one hand, and the respective sites of the AHs specified below, on the other hand, will be accordingly designated by $\mu$ and $v$. Parameters $\gamma, \mu$ and $v$ are supposed to take sufficiently small values (compared with the above-specified energy unit) so that these may be included in the first order Hamiltonian matrix $\boldsymbol{H}_{(1)}$.

Let the electron-donating subsystem $\mathrm{D}$ interact with the 1 st $\mathrm{AO}$ of the subset $\left\{\chi^{*}\right\}$, i.e. with $\chi_{1}^{*}$. Concerning the site of the electron-accepting orbital $\psi_{a}$, two cases may be distinguished: The first one coincides with the orbital $\psi_{a}$ being attached to an AO of the opposite subset $\left\{\chi^{\circ}\right\}$, e.g. $\chi_{1}^{o}$. The relevant first order Hamiltonian matrix will be denoted by $\boldsymbol{H}_{(1)}^{*_{0}}$. The second case refers to an interaction between the orbital $\psi_{a}$ and an $\mathrm{AO}$ of the same subset $\left\{\chi^{*}\right\}$, say $\chi_{j}^{*}$, where $j \neq 1$. The respective first order Hamiltonian matrix then acquires the superscript $* *$. The matrices concerned may be represented as follows

$$
\boldsymbol{H}_{(1)}^{*_{0}}=\left|\begin{array}{cccc}
\mathbf{0} & \boldsymbol{d}_{1} & \mathbf{0} & \mathbf{0} \\
\boldsymbol{d}_{1}^{+} & 0 & \gamma & \mathbf{0} \\
\mathbf{0} & \gamma & 0 & \boldsymbol{a}_{1} \\
\mathbf{0} & \mathbf{0} & \boldsymbol{a}_{1}^{+} & \mathbf{0}
\end{array}\right|, \quad \boldsymbol{H}_{(1)}^{* *}=\left|\begin{array}{cccc}
\mathbf{0} & \boldsymbol{d}_{1} & \boldsymbol{a}_{j} & \mathbf{0} \\
\boldsymbol{d}_{1}^{+} & 0 & \gamma & \mathbf{0} \\
\boldsymbol{a}_{j}^{+} & \gamma & 0 & \mathbf{0} \\
\mathbf{0} & \mathbf{0} & \mathbf{0} & \mathbf{0}
\end{array}\right|,
$$

where $\boldsymbol{d}_{1}$ and $\boldsymbol{a}_{j}$ are column-matrices containing nonzero elements $\mu$ and $v$ in the 1 st and jth positions, respectively, whilst $\boldsymbol{a}_{1}$ is a row-matrix, the first element of which coincides with $v$.

As already mentioned, the common Hückel type Hamiltonian matrix of AHs may be block-diagonalized non-perturbatively using an unitary transformation matrix 32. The same procedure may be straightforwardly extended to the matrix $\boldsymbol{H}_{(0)}$ of Eq. 1. The relevant transformation matrix $\boldsymbol{C}$ may be expressed either in terms of the principal matrix $\boldsymbol{R}$ or via its counterpart $\boldsymbol{Q}$, where

$$
\boldsymbol{R}=\left(\boldsymbol{B} \boldsymbol{B}^{+}\right)^{-1 / 2}, \quad \boldsymbol{Q}=\left(\boldsymbol{B}^{+} \boldsymbol{B}\right)^{-1 / 2}
$$

and

$$
R B=B Q
$$

(The positive-definite square root is assumed to be chosen in Eq. 3). As for instance, the $\boldsymbol{Q}$-representation concerned takes the form

$$
\boldsymbol{C}=\frac{1}{\sqrt{2}}\left|\begin{array}{cccc}
\boldsymbol{I} & \mathbf{0} & \mathbf{0} & \boldsymbol{B} \boldsymbol{Q} \\
\mathbf{0} & \sqrt{2} & 0 & \mathbf{0} \\
\mathbf{0} & 0 & \sqrt{2} & \mathbf{0} \\
\boldsymbol{Q} \boldsymbol{B}^{+} & \mathbf{0} & \mathbf{0} & -\boldsymbol{I}
\end{array}\right|
$$

where $I$ here and below stands for the unit matrix. Besides, the unitarity condition for the matrix $\boldsymbol{C}$ yields the following useful relations

$$
R B B^{+} R=Q B^{+} B Q=B Q Q B^{+}=B^{+} R R B=I .
$$

Finally, the relevant transformed matrix $\left(\tilde{\boldsymbol{H}}_{(0)}\right)$ contains two non-zero eigenblocks corresponding to subsets $\left\{\chi^{*}\right\}$ and $\left\{\chi^{\circ}\right\}$ and a $2 \times 2$-dimensional zero block referring to orbitals $\psi_{d}$ and $\psi_{a}$, i.e.

$$
\tilde{\boldsymbol{H}}_{(0)}=\boldsymbol{C}^{+} \boldsymbol{H}_{(0)} \boldsymbol{C}=\left|\begin{array}{cccc}
\boldsymbol{E}_{(+)} & \mathbf{0} & \mathbf{0} & \mathbf{0} \\
\mathbf{0} & 0 & 0 & \mathbf{0} \\
\mathbf{0} & 0 & 0 & \mathbf{0} \\
\mathbf{0} & \mathbf{0} & \mathbf{0} & -\boldsymbol{E}_{(-)}
\end{array}\right|,
$$

where $\boldsymbol{E}_{(+)}$and $\boldsymbol{E}_{(-)}$are the following $n \times n-$ dimensional submatrices

$$
\boldsymbol{E}_{(+)}=\boldsymbol{R}^{-1}=\left(\boldsymbol{B} \boldsymbol{B}^{+}\right)^{1 / 2}, \quad \boldsymbol{E}_{(-)}=\boldsymbol{Q}^{-1}=\left(\boldsymbol{B}^{+} \boldsymbol{B}\right)^{1 / 2}
$$

and the minus sign in front of $\boldsymbol{E}_{(-)}$is introduced for convenience.

It is evident that the above-described transformation may also be applied to first order matrices of 
Eq. 2. For $\boldsymbol{H}_{(1)}^{*_{0}}$ and $\boldsymbol{H}_{(1)}^{* *}$, we accordingly obtain

$$
\begin{aligned}
\tilde{\boldsymbol{H}}_{(1)}^{*_{0}} & =\boldsymbol{C}^{+} \boldsymbol{H}_{(1)}^{* o} \boldsymbol{C}= \\
& =\frac{1}{\sqrt{2}}\left|\begin{array}{cccc}
\mathbf{0} & \boldsymbol{d}_{1} & \boldsymbol{B} \boldsymbol{Q} \boldsymbol{a}_{1}^{+} & \mathbf{0} \\
\boldsymbol{d}_{1}^{+} & 0 & \sqrt{2} \gamma & \boldsymbol{d}_{1}^{+} \boldsymbol{B} \boldsymbol{Q} \\
\boldsymbol{a}_{1} \boldsymbol{Q} \boldsymbol{B}^{+} & \sqrt{2} \gamma & 0 & -\boldsymbol{a}_{1} \\
\mathbf{0} & \boldsymbol{Q} \boldsymbol{B}^{+} \boldsymbol{d}_{1} & -\boldsymbol{a}_{1}^{+} & \mathbf{0}
\end{array}\right|, \\
\tilde{\boldsymbol{H}}_{(1)}^{* *} & =\boldsymbol{C}^{+} \boldsymbol{H}_{(1)}^{* *} \boldsymbol{C}=\frac{1}{\sqrt{2}}\left|\begin{array}{cccc}
\mathbf{0} & \boldsymbol{d}_{1} & \boldsymbol{a}_{j} & \mathbf{0} \\
\boldsymbol{d}_{1}^{+} & 0 & \sqrt{2} \gamma & \boldsymbol{d}_{1}^{+} \boldsymbol{B} \boldsymbol{Q} \\
\boldsymbol{a}_{j}^{+} & \sqrt{2} \gamma & 0 & \boldsymbol{a}_{j}^{+} \boldsymbol{B} \boldsymbol{Q} \\
\mathbf{0} & \boldsymbol{Q} \boldsymbol{B}^{+} \boldsymbol{d}_{1} & \boldsymbol{Q} \boldsymbol{B}^{+} \boldsymbol{a}_{j} & \mathbf{0}
\end{array}\right| .
\end{aligned}
$$

Moreover, the respective total transformed Hamiltonian matrices of our systems D-AH-A defined as follows

$$
\tilde{\boldsymbol{H}}^{*_{o}}=\tilde{\boldsymbol{H}}_{(0)}+\tilde{\boldsymbol{H}}_{(1)}^{*_{o}}, \quad \tilde{\boldsymbol{H}}^{* *}=\tilde{\boldsymbol{H}}_{(0)}+\tilde{\boldsymbol{H}}_{(1)}^{* *}
$$

are nearly block-diagonal, i.e. these contain off-diagonal blocks of first order magnitude only. Hence, the perturbative methodology of Refs. 50-53 may be applied to complete the overall block-diagonalization procedure. The essence of this methodology consists in searching both for the appropriate transformation matrix $\boldsymbol{T}$ and for eigenblocks of the matrix concerned in the form of a power series (e.g. the matrix $\boldsymbol{T}$ is expressed as a sum over parameter $k$ of various increments $\boldsymbol{T}_{(k)}$, where $k$ stands for the order of the given term with respect to entire submatrices of the initial first order (perturbation) matrix).

To overview the principal formulae of this approach, let us abandon for a moment the particular matrices of Eqs. 7-11 and consider the more general case of $N$ weakly-interacting subsets of basis functions.

Suppose that our zero order Hamiltonian matrix takes a block-diagonal form like that of Eq. 7 and contains $N$ eigenblocks $\boldsymbol{E}_{(0) r}, r=1,2 \ldots N$ in its diagonal positions. Further, let $\boldsymbol{V}$ stand for a certain perturbation matrix that consists of blocks $\boldsymbol{V}_{r s}$ of respective dimensions, where $r$ and $s$ also embrace numbers from 1 to $N$. Finally, the transformation matrix $\boldsymbol{T}$ under our search also is assumed to be accordingly divided into blocks $\boldsymbol{T}_{r s}(r, s=1 \ldots N)$. Besides, the subsets of basis functions are supposed here to be separated by substantial energy gaps $v s$. the intersubset interactions contained within blocks $\boldsymbol{V}_{r s}$.

Under the above-enumerated conditions, the first and the second order corrections to an individual eigenblock $\boldsymbol{E}_{(0) r}$ were shown to take the form ${ }^{51-53}$

$$
\boldsymbol{E}_{(1) r}=\boldsymbol{V}_{r r}
$$

and

$$
\boldsymbol{E}_{(2) r}=\frac{1}{2} \sum_{s=1}^{N}\left(1-\delta_{r s}\right)\left(\boldsymbol{V}_{s r}^{+} \boldsymbol{T}_{(1) s r}+\boldsymbol{T}_{(1) s r}^{+} \boldsymbol{V}_{s r}\right),
$$

respectively, where $\boldsymbol{V}_{s r}^{+}=\boldsymbol{V}_{r s}$, but $\boldsymbol{T}_{(1) s r}^{+}=-\boldsymbol{T}_{(1) r s}$ as established previously ${ }^{50}$. Submatrices $\left(\boldsymbol{T}_{(1) s r}\right)$ of the first order member $\left(\boldsymbol{T}_{(1)}\right)$ of the power series for the transformation matrix $\boldsymbol{T}$, in turn, are conditioned by matrix equations, viz.

$$
\boldsymbol{E}_{(0) r} \boldsymbol{T}_{(1) r s}-\boldsymbol{T}_{(1) r s} \boldsymbol{E}_{(0) s}+\boldsymbol{V}_{r s}=\mathbf{0}
$$

Let us return again to our specific matrices $\tilde{\boldsymbol{H}}^{* o}$ and $\tilde{\boldsymbol{H}}^{* *}$ defined by Eqs. 7-11 and apply the formulae of Eqs. $12-14$ to block-diagonalize them. Orbitals of external groups D and A (i.e. $\psi_{d}$ and $\psi_{a}$ ) will be assumed now to make up the second subset, whilst the first and the third subsets correspondingly coincide with $\left\{\chi^{*}\right\}$ and $\left\{\chi^{\circ}\right\}$. From Eqs. 7, 9, 10 and 12 , we then obtain

$$
\begin{aligned}
& \boldsymbol{E}_{(0) 1}=\boldsymbol{E}_{(+)}, \quad \boldsymbol{E}_{(0) 2}=\mathbf{0}, \quad \boldsymbol{E}_{(0) 3}=-\boldsymbol{E}_{(-)}, \\
& \boldsymbol{E}_{(1) 1}=\boldsymbol{E}_{(1) 3}=\mathbf{0}, \quad \boldsymbol{E}_{(1) 2}=\left|\begin{array}{ll}
0 & \gamma \\
\gamma & 0
\end{array}\right| .
\end{aligned}
$$

Thus, the first non-zero member of the power series for the eigenblock under our interest (i.e. for $\boldsymbol{E}_{2}$ ) coincides with the $2 \times 2$-dimensional matrix $\boldsymbol{E}_{(1) 2}$. The latter contains the direct (through-space) interaction $(\gamma)$ between the external orbitals $\psi_{d}$ and $\psi_{a}$ in its off-diagonal positions in accordance with expectation. The next (second order) member of the same series $\left(\boldsymbol{E}_{(2) 2}\right)$ follows from Eq. 13. After invoking the equality $\boldsymbol{V}_{13}=\mathbf{0}$ following from Eqs. 9 and 10, we see that the expression concerned contains submatrices $\boldsymbol{T}_{(1) 12}$ and $\boldsymbol{T}_{(1) 23}$ only, viz.

$$
\boldsymbol{E}_{(2) 2}=\frac{1}{2}\left(\boldsymbol{V}_{12}^{+} \boldsymbol{T}_{(1) 12}+\boldsymbol{T}_{(1) 12}^{+} \boldsymbol{V}_{12}-\boldsymbol{V}_{23} \boldsymbol{T}_{(1) 23}^{+}-\boldsymbol{T}_{(1) 23} \boldsymbol{V}_{23}^{+}\right)
$$

where the relation $\boldsymbol{T}_{(1) s r}^{+}=-\boldsymbol{T}_{(1) r s}$ is also invoked. Further, coincidence of the zero order eigenblock $\boldsymbol{E}_{(0) 2}$ with the zero matrix (see the second relation of Eq. 15) allows matrix equations of Eq. 14 conditioning the blocks $\boldsymbol{T}_{(1) 12}$ and $\boldsymbol{T}_{(1) 23}$ to be solved algebraically. After substituting $\boldsymbol{R}^{-1}$ and $\boldsymbol{Q}^{-1}$ for $\boldsymbol{E}_{(0) 1}$ and $\boldsymbol{E}_{(0) 3}$, respectively, on the basis of Eq. 8 we obtain

$$
\boldsymbol{T}_{(1) 12}=-\boldsymbol{R} \boldsymbol{V}_{12}, \boldsymbol{T}_{(1) 23}=-\boldsymbol{V}_{23} \boldsymbol{Q}
$$

Now, no more is required than to take the submatrices $\boldsymbol{V}_{12}$ and $\boldsymbol{V}_{23}$ from Eqs. 9 and 10 and to substitute them 
into Eqs. 16 and 17. To distinguish between $\boldsymbol{V}_{12}$ (and $\left.\boldsymbol{V}_{23}\right)$ originating from matrices $\tilde{\boldsymbol{H}}^{* o}$ and $\tilde{\boldsymbol{H}}^{* *}$, the supercripts $*^{0}$ and $* *$ will accordingly be used. Analogous notations will also be added to the resulting second order corrections $\boldsymbol{E}_{(2) 2}$.

Let us dwell first on the perturbation matrix $\tilde{\boldsymbol{H}}^{* o}$ of Eq. 9. The relevant submatrices $\boldsymbol{V}_{12}^{*_{0}}$ and $\boldsymbol{V}_{23}^{*_{0}}$ take the form

$$
\boldsymbol{V}_{12}^{*_{0}}=\frac{1}{\sqrt{2}}\left|\begin{array}{ll}
d_{1} & \boldsymbol{B} \boldsymbol{Q \boldsymbol { a } _ { 1 } ^ { + }}
\end{array}\right|, \quad \boldsymbol{V}_{23}^{*_{\mathrm{o}}}=\frac{1}{\sqrt{2}}\left|\begin{array}{c}
\boldsymbol{d}_{1}^{+} \boldsymbol{B} \boldsymbol{Q} \\
-\boldsymbol{a}_{1}
\end{array}\right| .
$$

Let us recall now that the only off-diagonal element $\boldsymbol{E}_{(2) 2, d a}^{*_{0}}$ of the second order member $\boldsymbol{E}_{(2) 2}^{*_{\mathrm{o}}}$ of the power series for the eigenblock $\boldsymbol{E}_{2}^{*_{\mathrm{o}}}$ is expected to represent the alternant-hydrocarbon-assisted interaction between the external orbitals $\psi_{d}$ and $\psi_{a}$. The relevant expression is as follows

$$
\boldsymbol{E}_{(2) 2, d a}^{*_{0}}=-\boldsymbol{a}_{1} \boldsymbol{Q}^{2} \boldsymbol{B}^{+} \boldsymbol{d}_{1}=-\boldsymbol{a}_{1} \boldsymbol{B}^{-1} \boldsymbol{d}_{1},
$$

where Eq. 6 is additionally invoked when deriving the last relation. It is seen that the above formula for $\boldsymbol{E}_{(2) 2, d a}^{*_{0}}$ contains the inverse counterpart of the matrix $\boldsymbol{B}$ and thereby depends on the characteristics of the bridge. Thus, the indirect (bridge-assisted) nature of the interaction represented by the second order element $\boldsymbol{E}_{(2) 2, d a}^{*_{\mathrm{o}}}$ is obvious. More information about the same element follows from Eq. 19 after invoking specific constitutions of matrices $\boldsymbol{a}_{1}$ and $\boldsymbol{d}_{1}$ (see the discussion just after Eq. 2). We may then conclude the effective bridge-assisted interaction to be actually proportional to the single element $\left(\boldsymbol{B}^{-1}\right)_{11}$ of the matrix $\boldsymbol{B}^{-1}$ embracing the AOs $\chi_{1}^{*}$ and $\chi_{1}^{o}$, the external orbitals $\psi_{d}$ and $\psi_{a}$ that are attached to. [The meaning of the minus sign of Eq. 19 will be clarified in the last Section]. It also deserves adding here that diagonal elements $\left(\boldsymbol{E}_{(2) 2, d d}^{*_{0}}\right.$ and $\left.\boldsymbol{E}_{(2) 2, a a}^{*_{0}}\right)$ of the same second order correction $\boldsymbol{E}_{(2) 2}^{*_{0}}$ to the eigenblock $\boldsymbol{E}_{2}^{*_{0}}$ take zero values. This implies that relative positions of energy levels of external orbitals $\psi_{d}$ and $\psi_{a}$ are not influenced by the presence of the bridge.

Application of an analogous procedure to the Hamiltonian matrix $\tilde{\boldsymbol{H}}^{* *}$ of Eq. 10 shows that increments of the right-hand side of Eq. 16 cancel out one another in this case. As a result, the block $\boldsymbol{E}_{(2) 2}^{* *}$ coincides with the zero matrix. This implies that the external orbitals $\psi_{d}$ and $\psi_{a}$ do not interact indirectly if these are attached to AOs of the same subset of the bridge.

In summary, a general selection rule follows from the above conclusions for matrix elements (effective resonance parameters) of indirect interactions of orbitals of external groups D and A via an $\mathrm{AH}$, viz. the elements concerned take non-zero values if the groups D and A are attached to AOs of the bridge belonging to different subsets and vanish otherwise. Qualitatively, a similar rule resulted also from analysis of phases and amplitudes of molecular orbitals (MOs) of particular aromatic hydrocarbons ${ }^{10}$ and has been traced back to symmetry properties of the MOs. Finally, the rule under discussion proves to be in agreement with experimental findings concerning the ET properties of benzene. ${ }^{10,20}$ Besides, the analogy is noteworthy between the abovederived selection rule and that governing the electronic structures of the diradical even AHs. ${ }^{56,57}$ Indeed, the radical centers ,do not interact one with another" when bound to AOs of a classical even $\mathrm{AH}$ of the same subset and, consequently, the relevant diradical $\mathrm{AH}$ is characterized by two non-bonding molecular orbitals (NBMOs), e.g. in meta-quinodimethane. ${ }^{57}$ By contrast, diradicals contain no NBMOs, if the radical centers are attached to AOs of opposite subsets of an even $\mathrm{AH}$.

Let us turn again to Eq. 19 and recall that the total common AM $(\boldsymbol{A})$ of graphs of AHs and its inverse counterpart $\left(\boldsymbol{A}^{-1}\right)$ take the following forms

$$
\boldsymbol{A}=\left|\begin{array}{cc}
\mathbf{0} & \boldsymbol{B} \\
\boldsymbol{B}^{+} & \mathbf{0}
\end{array}\right|, \quad \boldsymbol{A}^{-1}=\left|\begin{array}{cc}
\mathbf{0} & \left(\boldsymbol{B}^{+}\right)^{-1} \\
\boldsymbol{B}^{-1} & \mathbf{0}
\end{array}\right|
$$

and contain non-zero blocks in the off-diagonal (intersubset) positions only (see also Eq. 1 and the discussion nearby). This implies the above-specified decisive elements of the submatrix $\boldsymbol{B}^{-1}$ to coincide with those of the total IAM $\left(\boldsymbol{A}^{-1}\right)$ between AOs of different subsets. Moreover, parallelism is evident between the constitution of the IAM $\boldsymbol{A}^{-1}$ of Eq. 20 and the abovederived selection rule for resonance parameters of indirect interactions between external orbitals $\psi_{d}$ and $\psi_{a}$. Indeed, vanishing interactions correspond to zero intrasubset blocks of the total IAM $\boldsymbol{A}^{-1}$, whereas the non-zero interactions are proportional to respective elements of the non-zero submatrix $\boldsymbol{B}^{-1}$. Hence, the above-derived selection rule may be traced back to the constitution of the IAM $\boldsymbol{A}^{-1}$ of Eq. 20.

Before finishing this section, the interrelation deserves mentioning between IAM elements and the respective Ruedenberg or Pauling bond orders. ${ }^{58,59}$ In our context, this relation indicates that the stronger is the bond order between AOs $\chi_{1}^{*}$ and $\chi_{1}^{o}$ of our $\mathrm{AH}$, the more efficient is the effective indirect interaction between the external groups D and A attached to these AOs.

\section{EXPRESSIONS FOR INVERSE ADJACENCY MATRICES OF VARIOUS TYPES OF AHS}

The principal result of the previous Section offers a new field of application of the inverse AMs (IAMs) of 
molecular graphs, namely qualitative studies of bridgeassisted ET processes via AHs. Employment of the adjacency matrix inversion for investigation of spectral properties of graphs with zero eigenvalues representing polymethine dyes ${ }^{60}$ may be mentioned here as a related achievement. Moreover, IAMs were shown to play an important role in establishing relations between the simplest versions of the MO and VB theories. ${ }^{61,62}$ The above-enumerated points stimulate an interest in properties of IAMs of AHs addresssed in this Section.

Let us note at first that several approaches are known $^{63}$ for derivation of IAMs in general. Accordingly, distinct interpretations follow for IAM elements in terms of peculiarities of the initial graph. As for instance, elements $\left(\boldsymbol{A}^{-1}\right)_{i j}$ have been related to certain valence structures of particular skeletal fragments, ${ }^{61,62}$ to the total number of pathways between vertices $i$ and $j{ }^{63}$ to coefficients of the secular polynomial of the initial graph ${ }^{64}$ etc. As already mentioned, the NCRSPT employed in the previous Section deals with entire blocks of initial matrices. To preserve the same methodology, we will invoke below a less popular general expression for the inverse counterpart of a square matrix in terms of its submatrices (blocks) known as the Frobenius formula. ${ }^{65}$

Let the AH under study consist of two evenmembered fragments I and II. It is evident that this partition is equivalent to distinguishing two subgraphs in the respective graph. As a result, the subsets of $2 p_{z}$ AOs of the previous Section (i.e. $\left\{\chi^{*}\right\}$ and $\left.\left\{\chi^{\circ}\right\}\right)$ may be further subdivided into four parts $\left\{\chi_{I}^{*}\right\},\left\{\chi_{I I}^{*}\right\},\left\{\chi_{I}^{o}\right\}$ and $\left\{\chi_{I I}^{o}\right\}$. Accordingly, the former matrix $\boldsymbol{B}$ may be represented in terms of four submatrices, viz.

$$
\boldsymbol{B}=\left|\begin{array}{cc}
\boldsymbol{B}_{\mathrm{I}} & \boldsymbol{K} \\
\boldsymbol{L} & \boldsymbol{B}_{\mathrm{II}}
\end{array}\right|,
$$

where $\boldsymbol{B}_{\mathrm{I}}$ and $\boldsymbol{B}_{\mathrm{II}}$ now refer to individual fragments (subgraphs) I and II, respectively. These intrafragmental blocks will be called the primary AM blocks for reasons clarified below. Meanwhile, the remaining submatrices $\boldsymbol{K}$ and $\boldsymbol{L}$ contain elements of interfragmental nature. It deserves a separate mentioning that the block $\boldsymbol{K}$ consists of elements connecting the subsets $\left\{\chi_{I}^{*}\right\}$ and $\left\{\chi_{I I}^{o}\right\}$, whereas $\boldsymbol{L}$ embraces the interfragmental interactions between orbitals belonging to $\left\{\chi_{I I}^{*}\right\}$ and $\left\{\chi_{I}^{o}\right\}$.

Let us turn now to the Frobenius formula. ${ }^{65}$ Under the above-specified partition of the total basis set of $2 p_{z}$ AOs, this formula yields an expression for the inverse counterpart $\boldsymbol{B}^{-1}$ of the matrix $\boldsymbol{B}$ of Eq. 21 in terms of intra- and interfragmental blocks of the latter, viz.

$$
\boldsymbol{B}^{-1}=\left|\begin{array}{cc}
\boldsymbol{B}_{\mathrm{I}}^{-1}+\boldsymbol{B}_{\mathrm{I}}^{-1} \boldsymbol{K} \boldsymbol{H}_{\mathrm{II}}^{-1} \boldsymbol{L} \boldsymbol{B}_{\mathrm{I}}^{-1} & -\boldsymbol{B}_{\mathrm{I}}^{-1} \boldsymbol{K} \boldsymbol{H}_{\mathrm{II}}^{-1} \\
-\boldsymbol{H}_{\mathrm{II}}^{-1} \boldsymbol{L} \boldsymbol{B}_{\mathrm{I}}^{-1} & \boldsymbol{H}_{\mathrm{II}}^{-1}
\end{array}\right|,
$$

where

$$
\boldsymbol{H}_{\mathrm{II}}=\boldsymbol{B}_{\mathrm{II}}-\boldsymbol{L} \boldsymbol{B}_{\mathrm{I}}^{-1} \boldsymbol{K} .
$$

Submatrices $\boldsymbol{B}_{\mathrm{I}}^{-1}$ and $\boldsymbol{B}_{\mathrm{II}}^{-1}$ evidently coincide with primary IAM blocks of respective fragments [Assumptions that $\boldsymbol{B}_{\mathrm{I}}$ and $\boldsymbol{B}_{\mathrm{II}}$ are square submatrices and $\operatorname{Det} \boldsymbol{B}_{\mathrm{I}} \neq 0$ underly the relation of Eq. 22]. It is also seen that the second term of the right-hand side of Eq. 23 contains both the IAM block of the first fragment $\left(\boldsymbol{B}_{I}^{-1}\right)$ and the interfragmental blocks $\boldsymbol{K}$ and $\boldsymbol{L}$ and thereby represents a certain environment-determined correction to the primary AM block of the second fragment $\left(\boldsymbol{B}_{\mathrm{II}}\right)$. Hence, the matrix $\boldsymbol{H}_{\mathrm{II}}$ may be interpreted as the adjusted AM block of the above-specified fragment (subgraph), whereas $\boldsymbol{H}_{\mathrm{II}}^{-1}$ stands for the relevant inverse adjusted AM block.

Let us turn now to some particular cases of Eq. 22. Let us assume first that our AM block $\boldsymbol{B}$ of Eq. 21 contains a zero off-diagonal submatrix, e.g. $\boldsymbol{L}=\mathbf{0}$. This implies that interactions between subsets $\left\{\chi_{I I}^{*}\right\}$ and $\left\{\chi_{I}^{o}\right\}$ take zero values. The term , an one-sided interfragmental interaction" seems to describe this particular case. It is noteworthy that numerous popular AHs meet the above condition, e.g. composite hydrocarbons (R-R')

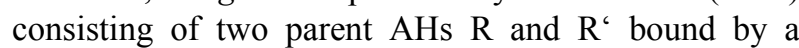
single bond (such as biphenyl). Thus, let us consider this particular case in more detail.

First of all, coincidence between matrices $\boldsymbol{B}_{\mathrm{II}}$ and $\boldsymbol{H}_{\text {II }}$ follows immediately from Eq. 23. Hence, the AM block of the second fragment undergoes no adjustment in the case of the one-sided interfragmental interaction. Consequently, Eq. 22 yields the following result

$$
\boldsymbol{B}^{-1}=\left|\begin{array}{cc}
\boldsymbol{B}_{\mathrm{I}}^{-1} & -\boldsymbol{B}_{\mathrm{I}}^{-1} \boldsymbol{K} \boldsymbol{B}_{\mathrm{II}}^{-1} \\
\mathbf{0} & \boldsymbol{B}_{\mathrm{II}}^{-1}
\end{array}\right| .
$$

It is seen that the primary IAM blocks of respective fragments (i.e. $\boldsymbol{B}_{\mathrm{I}}^{-1}$ and $\boldsymbol{B}_{\mathrm{II}}^{-1}$ ) take the diagonal positions in the above-exhibited matrix. This implies the intrafragmental IAM elements to be transferable from isolated fragments to the composite hydrocarbon. Furthermore, equation (24) yields an additional selection rule for IAM elements of the interfragmental nature, viz. these elements vanish just for pairs of AOs belonging to mutually-interacting subsets $\left\{\chi_{I}^{*}\right\}$ and $\left\{\chi_{I I}^{o}\right\}$. Meanwhile, non-zero values follow from Eq. 24 for IAM elements between AOs of non-interacting subsets $\left\{\chi_{I I}^{*}\right\}$ and $\left\{\chi_{I}^{o}\right\}$. [Note that subsets $\left\{\chi_{I}^{*}\right\}$ and $\left\{\chi_{I I}^{*}\right\}$ correspond to rows of the matrix $\boldsymbol{B}$ and to columns of $\left.\boldsymbol{B}^{-1}\right]$. Analogous transferability and selection rules evidently follow for ET properties of AHs characterized by an one-sided interfragmental interaction.

The particular result of Eq. 24 and the above conclusions may be easily extended to the case of 


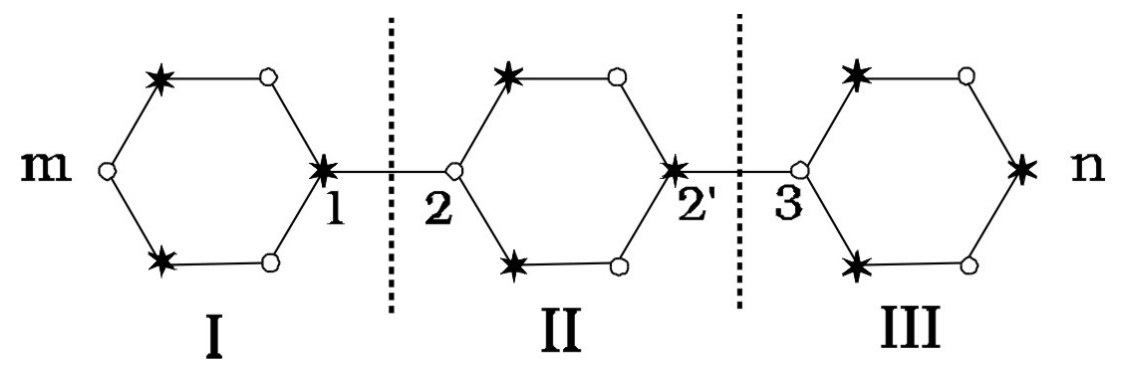

Figure 1. Triphenyl as an example of a chain-like AH, the neighboring fragments of which are characterized by one-sided interfragmental interactions.

several fragments undergoing one-sided interfragmental interactions. Let us assume for example that our matrix $\boldsymbol{B}$ is expressible as follows

$$
\boldsymbol{B}=\left|\begin{array}{ccc}
\boldsymbol{B}_{\mathrm{I}} & \boldsymbol{K} & \mathbf{0} \\
\mathbf{0} & \boldsymbol{B}_{\mathrm{II}} & \boldsymbol{M} \\
\mathbf{0} & \mathbf{0} & \boldsymbol{B}_{\mathrm{III}}
\end{array}\right|
$$

The relevant IAM block then takes the form

$$
\boldsymbol{B}^{-1}=\left|\begin{array}{ccc}
\boldsymbol{B}_{\mathrm{I}}^{-1} & -\boldsymbol{B}_{\mathrm{I}}^{-1} \boldsymbol{K} \boldsymbol{B}_{\mathrm{II}}^{-1} & \boldsymbol{B}_{\mathrm{I}}^{-1} \boldsymbol{K} \boldsymbol{B}_{\mathrm{II}}^{-1} \boldsymbol{M} \boldsymbol{B}_{\mathrm{III}}^{-1} \\
\mathbf{0} & \boldsymbol{B}_{\mathrm{II}}^{-1} & -\boldsymbol{B}_{\mathrm{II}}^{-1} \boldsymbol{M} \boldsymbol{B}_{\mathrm{III}}^{-1} \\
\mathbf{0} & \mathbf{0} & \boldsymbol{B}_{\mathrm{III}}^{-1}
\end{array}\right| .
$$

It deserves emphasizing here that the signs in front of matrix products alternate within any row (column) of the matrix $\boldsymbol{B}^{-1}$. Accordingly, the non-zero off-diagonal block of the matrix $\boldsymbol{B}^{-1}$ connecting AOs of terminal fragments acquires a parity factor $(-1)^{N+1}$ in the case of an arbitrary number of fragments $(N)$.

Let us return again to the three-fragmental system underlying Eqs. 25 and 26 and assume in addition that each interfragmental block (viz. $\boldsymbol{K}$ and $\boldsymbol{M}$ ) contains only a single non-zero element, e.g. $\boldsymbol{K}_{12} \neq 0$ and $\boldsymbol{M}_{2^{\prime} 3} \neq 0$ as exemplified by the triphenyl system of Figure 1. For an IAM element $\left(\boldsymbol{B}^{-1}\right)_{m n}$ connecting the AOs $\chi_{I, m}^{o}$ and $\chi_{I I I, n}^{*}$, we then obtain

$$
\left(\boldsymbol{B}^{-1}\right)_{m n}=\left(\boldsymbol{B}_{\mathrm{I}}^{-1}\right)_{m 1} \boldsymbol{K}_{12}\left(\boldsymbol{B}_{\mathrm{II}}^{-1}\right)_{22}, \boldsymbol{M}_{2 ' 3}\left(\boldsymbol{B}_{\mathrm{III}}^{-1}\right)_{3 n}
$$

in accordance with the only pathway between the AOs concerned. It is seen that the above expression contains intrafragmental IAM elements $\left(\boldsymbol{B}_{\mathrm{I}}^{-1}\right)_{m 1}$, $\left(\boldsymbol{B}_{\mathrm{II}}^{-1}\right)_{22}$, and $\left(\boldsymbol{B}_{\mathrm{III}}^{-1}\right)_{3 n}$ along with the interfragmental resonance parameters. After extension of this result to the case of $N$ elementary fragments, the analogue of Eq. 27 also contains the parity factor $(-1)^{N+1}$ indicating alternation of signs of IAM elements under consideration. Thus, we actually obtain an extension of the perurbation-theory-based McConnell formula ${ }^{66}$ widely used when studying the ET reactions ${ }^{38,44-46,48,67}$ and of the so-called parity rule $e^{44,46,67}$ to the case of chain-like bridges consisting of $N$ even AHs playing the role of fragments. An additional discussion of the parity rule and of its extension is undertaken in the next section.

Let us now go back to Eqs. 22 and 23 and introduce the following notation for further convenience, viz.

$$
\begin{aligned}
& \boldsymbol{L} \boldsymbol{B}_{\mathrm{I}}^{-1} \boldsymbol{K}=\boldsymbol{S}_{\mathrm{II}}, \quad \boldsymbol{K} \boldsymbol{H}_{\mathrm{II}}^{-1} \boldsymbol{L}=\boldsymbol{Q}_{\mathrm{I}}, \\
& \boldsymbol{B}_{\mathrm{I}}^{-1} \boldsymbol{K} \boldsymbol{H}_{\mathrm{II}}^{-1} \boldsymbol{L} \boldsymbol{B}_{\mathrm{I}}^{-1} \equiv \boldsymbol{B}_{\mathrm{I}}^{-1} \boldsymbol{Q}_{\mathrm{I}} \boldsymbol{B}_{\mathrm{I}}^{-1}=\boldsymbol{P}_{\mathrm{I}}
\end{aligned}
$$

As discussed already, the matrix $S_{\text {II }}$ represents the role of the first fragment in the formation of the adjusted AM block of the second fragment $\left(\boldsymbol{H}_{\mathrm{II}}\right)$, whereas $\boldsymbol{P}_{\mathrm{I}}$ coincides with the correction to the primary IAM block of the first fragment (i.e. to $\boldsymbol{B}_{\mathrm{I}}^{-1}$ ) owing to the presence of the second one.

Let us consider the case of the simplest two-sided interfragmental interaction characterized by two nonzero resonance parameters, e.g. $\boldsymbol{K}_{12}$ and $\boldsymbol{L}_{2}$ ‘ ' as exemplified in Figure 2 (The remaining elements of submatrices $\boldsymbol{K}$ and $\boldsymbol{L}$ are assumed to take zero values). Let us also accept the usual equalities $\boldsymbol{K}_{12}=\boldsymbol{L}_{2^{\prime} 1^{\natural}}=1$. The relevant matrix $\boldsymbol{S}_{\mathrm{II}}$ then accordingly contains a single non-zero element $\boldsymbol{S}_{\mathrm{II}, 2{ }^{\prime} 2}$ as the first relation of Eq. 28 shows and this element coincides with $\left(\boldsymbol{B}_{\mathrm{I}}^{-1}\right)_{1 ` 1}$. Hence, the role of the first fragment in the formation of the adjusted AM block of the second one $\left(\boldsymbol{H}_{\mathrm{II}}\right)$ resolves

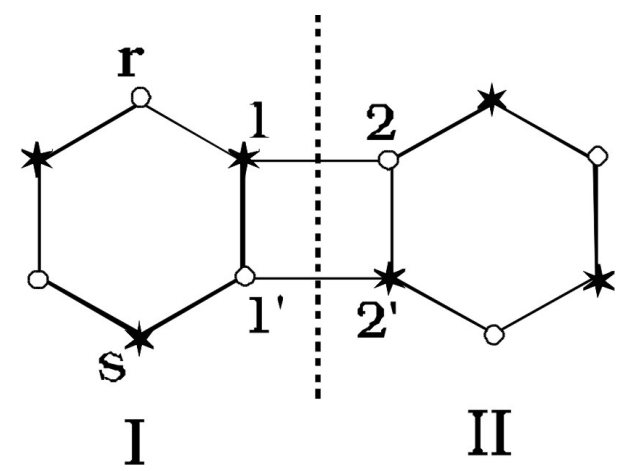

Figure 2. Biphenylene as an example of a two-sided interfragmental interaction. 
itself into a local perturbation embracing only two AOs $\chi_{I I, 2^{\prime}}^{*}$ and $\chi_{I I, 2}^{o}$ and determined by the initial IAM element of the first fragment $\left(\boldsymbol{B}_{I}^{-1}\right)_{1}$ ‘ between the sites $1^{\text {' }}$ and 1 undergoing the interfragmental interaction. It is evident that the above-concluded locality of perturbation is not preserved when passing to the IAM block $\boldsymbol{H}_{\mathrm{II}}^{-1}$. Nevertheless, the element $\left(\boldsymbol{B}_{\mathrm{I}}^{-1}\right)_{1^{`}{ }^{\prime} \text { still remains a }}$ certain criterion of the relative extent of the interfragmental influence in the given $\mathrm{AH}$ (see the next Section). Further, the relevant matrix $\boldsymbol{Q}_{\mathrm{I}}$ also contains $a$ single non-zero element $\boldsymbol{Q}_{\mathrm{I}, 11}$, coinciding with $\left(\boldsymbol{H}_{\mathrm{II}}^{-1}\right.$ )$_{22}$, i.e. with the actual (adjusted) IAM element between AOs $\chi_{I I, 2^{\prime}}^{*}$ and $\chi_{I I, 2}^{o}$. As a result, the element of the matrix $\boldsymbol{P}_{\mathrm{I}}$ referring to AOs $\chi_{I, s}^{*}$ and $\chi_{I, r}^{o}$ is expressible as follows

$$
\boldsymbol{P}_{\mathrm{I}, r s}=\left(\boldsymbol{B}_{\mathrm{I}}^{-1}\right)_{r 1}\left(\boldsymbol{H}_{\mathrm{II}}^{-1}\right)_{22}\left(\boldsymbol{B}_{\mathrm{I}}^{-1}\right)_{1^{\prime} s}
$$

It is seen that the correction $\left(\boldsymbol{P}_{\mathrm{I}, r s}\right)$ to the IAM element of the first fragment due to interfragmental interaction depends on two factors: The first one coincides with the actual IAM element between the

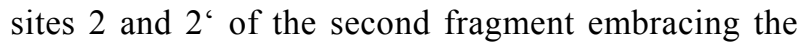
interfragmental bonds. The second factor is represented by the product of intrafragmental IAM elements $\left(\boldsymbol{B}_{\mathrm{I}}^{-1}\right)_{r 1}$ and $\left(\boldsymbol{B}_{\mathrm{I}}^{-1}\right)_{1}$ s. These elements evidently refer to an isolated fragment I and allow certain predictions to be made concerning the corrections $\boldsymbol{P}_{\mathrm{I}, r s}$. In particular, the largest value of the latter may be anticipated if the pairs of AOs $\chi_{I, 1}^{*}$ and $\chi_{I, r}^{o}$, as well as $\chi_{I, s}^{*}$ and $\chi_{I, 1^{\prime}}^{o}$ correspond to chemical bonds (as is the case in Figure 2).

Therefore, application of the Frobenius formula allows us to analyze the IAMs of various types of AHs and thereby the relevant ET properties in terms of fragments (subgraphs) and their interactions.

\section{CONSIDERATION OF SPECIFIC EXAMPLES}

Let us start with polyenes containing $N \mathrm{C}=\mathrm{C}$ bonds (Figure 3), the latter playing the role of fragments. It is evident that these chain-like systems are characterized by one-sided interfragmental interactions. In this connection, we may apply an expression like that of Eq. 26 to derive the relevant IAM block $\boldsymbol{B}^{-1}$.

First, let us consider the more general case of different resonance parameters of $\mathrm{C}=\mathrm{C}$ and $\mathrm{C}-\mathrm{C}$ bonds coinciding with 1 and $\sigma$, respectively, where $\sigma \leq 1$. We then obtain

$$
\boldsymbol{B}=\left|\begin{array}{ccccc}
1 & \sigma & 0 & 0 & \ldots \\
0 & 1 & \sigma & 0 & \ldots \\
0 & 0 & 1 & \sigma & \ldots \\
0 & 0 & 0 & 1 & \ldots \\
: & : & : & : & \ldots . .
\end{array}\right|, \boldsymbol{B}^{-1}=\left|\begin{array}{ccccc}
1 & -\sigma & \sigma^{2} & -\sigma^{3} & \ldots \\
0 & 1 & -\sigma & \sigma^{2} & \ldots \\
0 & 0 & 1 & -\sigma & \ldots \\
0 & 0 & 0 & 1 & \ldots \\
: & : & : & : & \ldots
\end{array}\right| .(30)
$$

It is seen that IAM elements of the largest absolute value (equal to 1 ) refer to intrafragmental $(\mathrm{C}=\mathrm{C})$ bonds in the polyene. Thus, the indirect interaction of the external groups D and A and thereby the consequent ET processes are predicted to be most efficient, if these groups are connected to sites $(1, N+1),(2, N+2)$, etc. Moreover, the above result embraces also the case of ethene $(N=1)$. Thus, the transferability rule for intrafragmental IAM elements (established in the previous Section) is now illustrated. Further, the IAM elements take zero values for interfragmental $(\mathrm{C}-\mathrm{C})$ bonds and the relevant indirect interactions thereby vanish in accordance with the selection rule based on Eq. 24 . Finally, extinction of the same elements follows from Eq. 30, when the distance between the AOs concerned increases. In particular, the third- and fifth-neighboring sites (e.g. $(2, N+1)$ and $(3, N+1)$, respectively) are correspondingly characterized by IAM elements equal to $-\sigma$ and $\sigma^{2}$. Alternation of signs of IAM elements when the number of mediating fragments grows is also among the implications of Eq. 30. It is also worth recalling here that the external groups $\mathrm{D}$ and $\mathrm{A}$ do not interact one with another if these are attached to AOs of the same subset, e.g. to the sites $(1,2),(N+1, N+2)$, etc. The particular case of uniform resonance parameters $(\sigma=1)$ deserves a separate mention. The point is that absolute values of bridge-assisted interactions are independent of the distance between the external groups D and A in this case. Nevertheless, alternation of signs of IAM elements still remains. The above-drawn conclusions may be illustrated using the butadiene molecule $(N=2)$ as an example: IAM elements equal to 1 and 0 refer to $C_{1}=C_{3}$ $\left(\mathrm{C}_{2}=\mathrm{C}_{4}\right)$ and $\mathrm{C}_{1}-\mathrm{C}_{4}$ bonds in this case. Meanwhile, the terminal sites $\left(C_{2}, C_{3}\right)$ are characterized by a negative IAM element equal to -1 .

Let us dwell now on the above-mentioned parity rule $^{44,46,67,68}$ and on its extensions following from Eqs. 26, 27 and 30. The original form of the rule ${ }^{68}$ concerns the dependence of the sign of the indirect interaction of the two external orbitals (and thereby of the relative order of the relevant two split energy levels ${ }^{46}$ ) upon the number of $\mathrm{C}-\mathrm{C}$ bonds in a saturated (sigma-bounded)

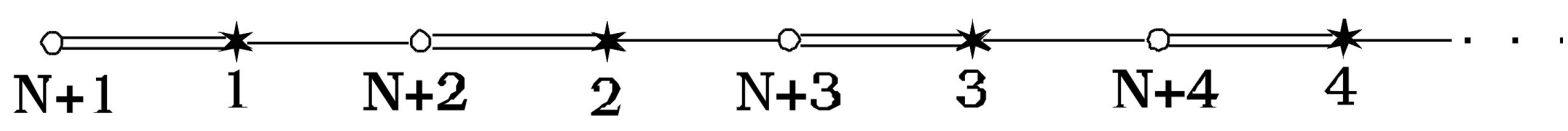

Figure 3. Numbering of $2 p_{z}$ AOs and/or carbon atoms in polyenes. 


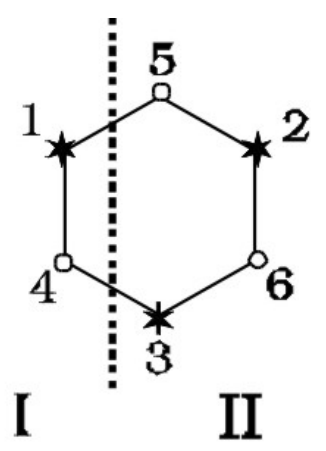

(a)

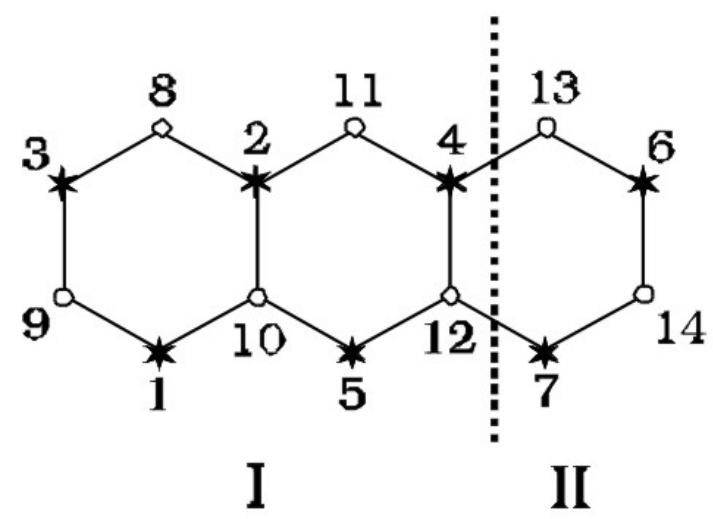

(c)

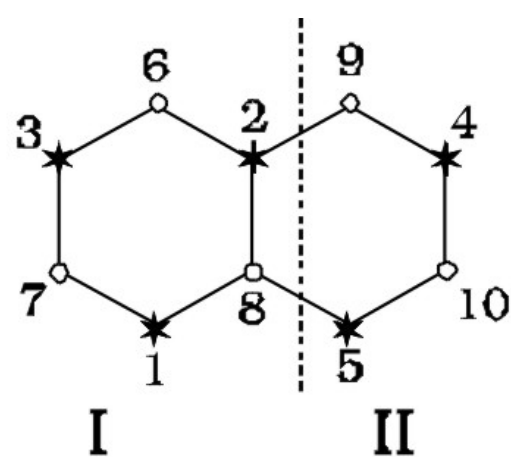

(b)

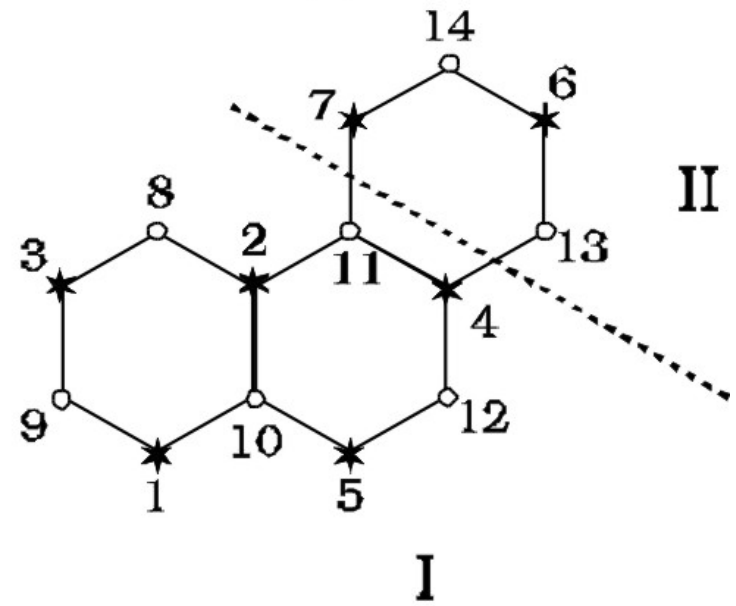

(d)

Figure 4. Formation of benzene (a), naphthalene (b), anthracene (c) and phenanthrene (d) as a result of a two-sided interaction of two fragments I and II, as well as numberings of the $2 \mathrm{p}_{\mathrm{z}} \mathrm{AOs}$ and/or carbon atoms.

bridge. The above-specified interaction was established to be a positive (negative) quantity for even (odd) numbers of intervening $\mathrm{C}-\mathrm{C}$ bonds (provided that all resonance parameters of the system are negative and a negative energy unit is used in addition). Because of the minus sign of Eq. 19, equation 30 now yields an extension of the same rule to the case of an unsaturated (polyene-like) bridge, where the number of $\mathrm{C}=\mathrm{C}$ bonds plays the role of the principal parameter. Accordingly, Eqs. 26 and 27 provide us with a generalization of the rule to bridges consisting of alternant fragments of arbitrary constitution.

The remaining part of this Section is devoted to aromatic hydrocarbons.

Let us start with the benzene molecule (Figure 4a). This classical AH may be conveniently considered as consisting of the ethene (I) and butadiene (II) fragments undergoing the simplest two-sided interfragmental interaction. Resonance parameters of all chemical bonds will be now taken equal to 1 . The relevant principal matrices are then as follows

$$
\begin{aligned}
& \boldsymbol{B}_{\mathrm{I}}=\boldsymbol{B}_{\mathrm{I}}^{-1}=1, \boldsymbol{B}_{\mathrm{II}}=\left|\begin{array}{ll}
1 & 1 \\
0 & 1
\end{array}\right|, \boldsymbol{B}_{\mathrm{II}}^{-1}=\left|\begin{array}{cc}
1 & -1 \\
0 & 1
\end{array}\right|, \boldsymbol{K}=\left|\begin{array}{ll}
1 & 0
\end{array}\right|, \\
& \boldsymbol{L}=\left|\begin{array}{l}
0 \\
1
\end{array}\right|, \boldsymbol{S}_{\mathrm{II}}=\left|\begin{array}{ll}
0 & 0 \\
1 & 0
\end{array}\right|, \boldsymbol{H}_{\mathrm{II}}=\left|\begin{array}{cc}
1 & 1 \\
-1 & 1
\end{array}\right|, \quad \boldsymbol{H}_{\mathrm{II}}^{-1}=\frac{1}{2}\left|\begin{array}{cc}
1 & -1 \\
1 & 1
\end{array}\right| .
\end{aligned}
$$

The initial IAM element of the 1st (ethene) fragment takes a significant value in this case, namely it coincides with 1, as the first relation of Eq. 31 indicates. This implies a comparatively large element $\left(\boldsymbol{B}_{\mathrm{I}}^{-1}\right)_{41}$, which is an analogue of $\left(\boldsymbol{B}_{\mathrm{I}}\right)_{1^{\circ} 1}$ of Figure 2 . As a result, the adjusted AM block of butadiene $\left(\boldsymbol{H}_{\mathrm{II}}\right)$ differs from the respective primary AM block $\left(\boldsymbol{B}_{\text {II }}\right)$ quite essentially (for comparison, Det $\boldsymbol{B}_{\mathrm{II}}=1$ and Det $\boldsymbol{H}_{\mathrm{II}}=2$ ) and thereby the interfragmental interaction is relatively strong in benzene. Such a conclusion evidently causes no surprise.

Elements of the inverse AM under our further interest are contained within the last matrix of Eq. 31, i.e. within $\boldsymbol{H}_{\mathrm{II}}^{-1}$. It is seen that elements equal to $1 / 2$ and $1 / 2$ refer to ortho and para positions of external groups, 
respectively. [Meta positions of benzene belong to the same subset and the relevant bridge-assisted interactions vanish (as discussed in the second Section)]. Comparison of these elements to those of butadiene shows that the above-concluded diene-like structure of the latter is now replaced by an aromatic one described by uniform IAM elements of an intermediate absolute value. Nevertheless, negative signs of IAM elements are preserved for para positions of benzene as it was the case with the terminal sites of butadiene.

Let us now consider an analogous scheme of formation of naphthalene, where the benzene molecule plays the role of the first fragment (Figure 4b). As discussed already, the IAM elements of benzene coincide with $1 / 2$ for any ortho position. The same evidently refers to atoms $\mathrm{C}_{2}$ and $\mathrm{C}_{8}$ undergoing the interaction with the second (butadiene) fragment. The abovementioned value (i.e. 1/2) makes up only a half of the relevant IAM element of ethene. Consequently, a suppressed interfragmental interaction and thereby a certain return to the diene-like pattern of IAM elements may be expected when passing from benzene to naphthalene.

To verify this anticipation, let us start with the matrix $S_{\text {II }}$ referring to the butadiene fragment (II) as previously. The only non-zero element of this matrix corresponds to the terminal sites 5 and 9 of this fragment and also coincides with $1 / 2$. The relevant adjusted AM block $\left(\boldsymbol{H}_{\mathrm{II}}\right)$ and its inverse counterpart are then as follows

$$
\boldsymbol{H}_{\mathrm{II}}=\left|\begin{array}{cc}
1 & 1 \\
-1 / 2 & 1
\end{array}\right|, \quad \boldsymbol{H}_{\mathrm{II}}^{-1}=\frac{1}{3}\left|\begin{array}{cc}
2 & -2 \\
1 & 2
\end{array}\right|
$$

Comparison of matrices $\boldsymbol{H}_{\mathrm{II}}$ of Eqs. 31 and 32 shows that the second one is much closer to the initial AM block of butadiene, the latter resulting from Eq. 30 under assumptions that $N=2$ and $\sigma=1$ (Note that Eq. 32 yields Det $\boldsymbol{H}_{\mathrm{II}}=3 / 2$ ). It is no surprise in this connection that distinct IAM elements (namely $2 / 3$ and $1 / 3$ ) follow from the matrix $\boldsymbol{H}_{\mathrm{II}}^{-1}$ of Eq. 32 for ortho positions $(4,9)$ $[(5,10)]$ and $(4,10)$, respectively. Accordingly, a more significant negative IAM element $(-2 / 3)$ corresponds to the para positions $(5,9)$. Finally, IAM elements of the interfragmental type easily follow from off-diagonal blocks of Eq. 22 after substituting the relevant matrix $\boldsymbol{H}_{\mathrm{II}}^{-1}$. In the case of naphthalene, these elements coincide either with $1 / 3$ or with $-1 / 3$. As for instance, we obtain $\left(\boldsymbol{B}^{-1}\right)_{56}=\left(\boldsymbol{B}^{-1}\right)_{47}=1 / 3$ and $\left(\boldsymbol{B}^{-1}\right)_{57}=\left(\boldsymbol{B}^{-1}\right)_{46}=-1 / 3$.

It is seen in summary that absolute values of nonzero IAM elements are not uniform in naphthalene, in contrast to benzene. Moreover, the largest IAM elements $( \pm 2 / 3)$ refer to sites $(4,9)$ and $(5,9)$. Thus, attachment of external groups D and A to just these sites is predicted to be most efficient in respect of the electron transfer. Meanwhile, the remaining pairs of carbon atoms (the AOs of which belong to different subsets) are expected to be of similar efficiencies, viz. $(4,10)$, $(5,7),(4,6),(5,6)$ and $(4,7)$. These results are in an excellent agreement with those following from both qualitative estimations of transmission probabilities on the basis of phase and amplitude of the HOMO and LUMO of naphthalene and computations of the same characteristics by means of density functional theory. ${ }^{10}$

The anthracene molecule is the next system we consider, wherein naphthalene and butadiene correspondingly play the role of the first and the second fragment. As already mentioned, an IAM element equal to $1 / 3$ refers to the bond of naphthalene that the butadiene fragment is subsequently attached to (the bond concerned coincides with $\mathrm{C}_{4}-\mathrm{C}_{10}$ of Figure $4 \mathrm{~b}$ and with $\mathrm{C}_{4}-$ $\mathrm{C}_{12}$ of Figure $4 \mathrm{c}$ ). Hence, a further extinction of the interfragmental interaction and thereby an even more diene-like pattern of IAM elements may be expected when passing to anthracene. Indeed, the matrix $\boldsymbol{H}_{\mathrm{II}}$ of the latter and its inverse counterpart are as follows

$$
\boldsymbol{H}_{\mathrm{II}}=\left|\begin{array}{cc}
1 & 1 \\
-1 / 3 & 1
\end{array}\right|, \quad \boldsymbol{H}_{\mathrm{II}}^{-1}=\frac{1}{4}\left|\begin{array}{cc}
3 & -3 \\
1 & 3
\end{array}\right|
$$

and yield Det $\boldsymbol{H}_{\mathrm{II}}=4 / 3$. Furthermore, IAM elements equal to $3 / 4$ and $1 / 4$ result from Eq. 33 for ortho positions inside the butadiene fragment of anthracene, viz. for $(6,13),[(7,14)]$ and $(6,14)$, respectively. In the case of para-positioned sites 7 and 13, the relevant IAM element is a negative quantity as previously and is equal to $-3 / 4$. It is also seen that the absolute value of this element grows compared with that of naphthalene. Thus, the overall pattern of the above-specified IAM elements becomes closer to that of an isolated butadiene. Elements inside the 1st (naphthalene) fragment of anthracene also are of interest. This especially refers to the sites 11 and 5 . The relevant expression easily follows from Eq. 29, viz.

$$
\boldsymbol{P}_{\mathrm{I}, 11,5}=\left(\boldsymbol{B}_{\mathrm{I}}^{-1}\right)_{11,4}\left(\boldsymbol{H}_{\mathrm{II}}^{-1}\right)_{13,7}\left(\boldsymbol{B}_{\mathrm{I}}^{-1}\right)_{12,5},
$$

where $\left(\boldsymbol{B}_{\mathrm{I}}^{-1}\right)_{11,4}$ coincides with $\left(\boldsymbol{B}_{\mathrm{I}}^{-1}\right)_{12,5}$ in addition. After substituting $2 / 3$ and $-3 / 4$ for $\left(\boldsymbol{B}_{I}^{-1}\right)_{11,4}$ and $\left(\boldsymbol{H}_{\mathrm{II}}^{-1}\right)_{13,7}$, respectively, we find that $\boldsymbol{P}_{\mathrm{I}, 11,5}$ equals to $1 / 3$. This correction should be subsequently added to the relevant primary value $\left(\boldsymbol{B}_{\mathrm{I}}^{-1}\right)_{11,5}=-2 / 3$ following from Eq. 32. Consequently, the IAM element concerned is equal to -1 and thereby proves to be of the largest absolute value in anthracene. The second largest one accordingly coincides with $\pm 3 / 4$ and refers to sites $(7,13)$ and $(7,14)$. These results also are in aggreement with those of Ref.10 concerning the transmission probabilities.

Let us finally consider an analogous scheme of formation of phenanthrene (Figure 4d). The decisive IAM element of the 1st (naphthalene) fragment now 
refers to the sites 4 and 9 of Figure $4 \mathrm{~b}$. The respective initial value of this element (equal to $2 / 3$ ) proves to be higher when compared with the relevant value in the case of anthracene $(1 / 3)$. This fact allows us to expect an increased interfragmental interaction in phenanthrene $v s$. that of anthracene. Accordingly, less distinct IAM elements are likely to correspond to ortho positions of respective terminal rings.

To verify these expectations, an analogue of Eq. 33 for phenanthrene is required. We accordingly obtain

$$
\boldsymbol{H}_{\mathrm{II}}=\left|\begin{array}{cc}
1 & 1 \\
-2 / 3 & 1
\end{array}\right|, \quad \boldsymbol{H}_{\mathrm{II}}^{-1}=\frac{1}{5}\left|\begin{array}{cc}
3 & -3 \\
2 & 3
\end{array}\right|,
$$

where Det $\boldsymbol{H}_{\mathrm{II}}=5 / 3$. The ortho positions of the second (butadiene) fragment of phenanthrene (namely 7,14 $(6,13)$ and 6,14$)$ are now correspondingly characterized by IAM elements equal to $3 / 5$ and $2 / 5$. These values evidently are more uniform as compared to their counterparts referring to anthracene (3/4 and $1 / 4$, respectively). The relevant para-positioned sites 7 and 13 are accordingly represented by a negative IAM element equal to $-3 / 5$. On the whole, a certain return to the aromatic (benzene-like) pattern of IAM elements may be concluded here.

For IAM elements inside the first (naphthalene) fragment of phenanthrene, an expression like that of Eq. 34 easily results from Eq. 29. As for instance, we obtain

$$
\boldsymbol{P}_{\mathrm{I}, 12,5}=\left(\boldsymbol{B}_{\mathrm{I}}^{-1}\right)_{12,4}\left(\boldsymbol{H}_{\mathrm{II}}^{-1}\right)_{13,7}\left(\boldsymbol{B}_{\mathrm{I}}^{-1}\right)_{11,5},
$$

where

$$
\left(\boldsymbol{B}_{\mathrm{I}}^{-1}\right)_{12,4}=1 / 3,\left(\boldsymbol{B}_{\mathrm{I}}^{-1}\right)_{11,5}=-2 / 3,\left(\boldsymbol{H}_{\mathrm{II}}^{-1}\right)_{13,7}=-3 / 5 .(37)
$$

Substituting Eq. 37 into Eq. 36 yields 2/15 for the correction $\boldsymbol{P}_{\mathrm{I}, 12,5}$. The latter should be subsequently added to the relevant primary value equal to $2 / 3$. The final result is then equal to $4 / 5$. This IAM element proves to be of the largest absolute value in phenanthrene, whilst $-3 / 5$ is the second largest one. The relevant transmission probabilities of Ref. 10 also comply with the aboveestablished order.

\section{CONCLUSIONS}

The principal achievements of the above study are:

1. Inverse adjacency matrices (IAMs) of graphs of C-skeletons of even AHs are established to play the decisive role in the formation of ET properties of these hydrocarbons. In particular, effective resonance parameters of indirect (bridgeassisted) interactions of orbitals of external groups D and A via an AH (determining the ET properties concerned) are shown to be proportional to individual elements of the relevant IAMs, namely to those between the sites (AOs) the groups D and A that they are attached to. On this basis, a common selection rule is formulated for the above-mentioned resonance parameters, viz. non-zero and zero values of the latter correspond to external groups attached to AOs of the AH belonging to different subsets $\left(\left\{\chi^{*}\right\}\right.$ and $\left\{\chi^{\circ}\right\}$ ) and to the same subset (either $\left\{\chi^{*}\right\}$ or $\left.\left\{\chi^{\circ}\right\}\right)$, respectively.

2. A general algebraic expression is derived and analyzed for the common IAM of all AHs in terms of intra- and interfragmental blocks of the initial AM. The nature of the interfragmental interaction then proves to be a criterion for distinguishing particular types of AHs and for formulating specific selection rules governing their ET properties. Hydrocarbons consisting of two or several even-membered fragments (subgraphs) undergoing the so-called one-sided interfragmental interaction may be mentioned here as an example. Transferability of ET properties of separate fragments after including the latter into a composite $\mathrm{AH}$ and an additional selection rule for interfragmental IAM elements are shown to be the most important distinctive features of these particular AHs.

3. The above-specified newly-derived expressions for IAMs are applied to reveal trends in ET properties within series of related AHs consisting of uniform fragments, e.g. of benzenoids. Regularities in the ET properties concerned are shown to originate from those in the relevant interfragmental interactions, the latter depending on the initial IAM element of an individual fragment between the sites bound to the remaining part of the given $\mathrm{AH}$.

On the whole, the above-enumerated results indicate that the molecular topology plays the decisive role in the formation of ET properties of AHs.

In respect of methodology, the novelty of the present study may be summarized as follows:

1. The approach applied is based on the nonperturbative block-diagonalization procedure for the common Hamiltonian matrix of $\mathrm{AHs}^{32}$. Consequently, the study embraces systems containing orbitals of uniform (or almost uniform) one-electron energies in contrast to the standard perturbation-theory-based methodologies (see e.g. Ref. 9 for an overview). In particular, coinciding one-electron energies are allowed here for orbitals of external groups and those of the bridge.

2. Application of the present approach to chain-like bridges consisting of even AHs as elementary 
fragments yields a generalization of the wellknown McConnell formula ${ }^{66}$. Moreover, the newly-derived expression for the effective resonance parameter of indirect interaction of external groups via the above-specified bridge exhibits alternation of sign with an increasing number of elementary fragments. The latter result implies an extension of the parity rule ${ }^{44,46}$ to the case of AH-containing bridges.

3. The results of the above study supplement the system of common rules governing the electronic structures of AHs with those representing the ET properties of these hydrocarbons. The general selection rule for effective resonance parameters of indirect interactions of external groups via an $\mathrm{AH}$ may be mentioned as the principal one. Specific rules governing the ET properties of bridges characterized by the so-called one-sided interfragmental interaction also deserves to be added.

\section{REFERENCES}

1. M. D. Newton, Adv. Chem. Phys. 106 (1999) 303-375.

2. C. C. Moser, J. M. Keske, K. Warncke, R. S. Farid, and P. L. Dutton, Nature (London) 355 (1992) 796-802.

3. S. Fukuzumi, H. Kotani, K. Ohkubo, S. Ogo, N. V. Tkachenko, and H. Lemmetyinen, J. Amer. Chem. Soc. 126 (2004) 1600-1601.

4. R. Pati and S. P. Karna, J. Chem. Phys. 115 (2001) 1703-1715.

5. J. W. Verhoeven, H. J. van Ramesdonk, M. M. Groeneveld, A. C. Benniston, and A. Harriman, Chem. Phys. Chem. 6 (2005) 2251-2260.

6. M. D. Newton and R. J. Cave, in: J. Jortner and M. A. Ratner (Eds.), Molecular Electronics, Blackwell Science, Oxford, 1977, p. 73.

7. N. J. Tao, Nature Nanotechnology 1 (2006) 173-181.

8. S. Kubatkin, A. Danilov, M. Hjort, J. Cornil, J. L. Bredas, N. Stuhr-Hansen, P. Hedegard, and T. Bjornholm, Nature 425 (2003) 698-701.

9. V. Gineityte, Lithuanian J. Phys. 42 (2002) 397-407.

10. K. Yoshizawa, T. Tada, and A. Staykov, J. Amer. Chem. Soc. 130 (2008) 9406-9413.

11. C. Chiorboli, M. A. J. Rodgers, and F. Scandola, J. Amer. Chem. Soc. 125 (2003) 483-491.

12. S. Creager, C.J. Yu, C. Bamdad, S. O`Connor, T. MacLean, E. Lam, Y. Chong, G.T. Olsen, J. Luo, M. Gozin, and J.F. Kayyem, J. Amer. Chem. Soc. 121 (1999) 1059-1064.

13. T. Kentaro, S. Azumi, Y. Tetsuro, A. Takuzo, Y. Kenichi, F. Mamoru, and I. Osamu, Chem. Lett. 35 (2006) 518-519.

14. J. M. F. Pinheiro Jr., P. H. R. Peixoto, and C. P. de Melo, Chem. Phys. Lett. 463 (2008) 172-177.

15. H. He, R. Pandey, R. Pati, and S. P. Karna, Phys. Rev. B73 (2006) 195311.

16. S.-H. Ke, H. U. Baranger, and W. Yang, J. Chem. Phys. 127 (2007) 144107.

17. Y. Noda, Sh. Noro, T. Akutagawa, and T. Nakamura, Phys. Rev. $B \mathbf{8 2}$ (2010) 205420.

18. A. Onipko and Y. Klymenko, J. Phys. Chem. A 102 (1998) 4246-4255.

19. S. Sitha and K. Bhanuprakash, J. Mol. Struct. (Theochem) 761 (2006) 31-38.

20. G. C. Solomon, C. Herrmann, J. Vura-Weis, M. R. Wasielewski, and M. A. Ratner, J. Amer. Chem. Soc. 132 (2010) 7887-7889.
21. C.A. Coulson, B.O'Leary and R. B. Mallion, Hückel Theory for Organic Chemistry, Academic Press, London, 1978.

22. N. Trinajstic in: G. A. Segal (Ed.), Semiempirical Methods of Electronic Structure Calculations, Part A, Techniques, Plenum Press, New York and London, 1977.

23. G. G. Hall, Proc. Roy. Soc. (London) A229 (1955) 251-259.

24. V. Gineityte, J. Mol. Struct. (Theochem) 487 (1999) 231-240.

25. C. A. Coulson and H. C. Longuet-Higgins, Proc. Roy. Soc. (London) A191 (1947) 39-60.

26. C. A. Coulson and H. C. Longuet-Higgins, Proc. Roy. Soc. (London) A192 (1947) 16-32.

27. C. A. Coulson and H. C. Longuet-Higgins, Proc. Roy. Soc. (London) A193 (1948) 447-464.

28. C. A. Coulson and H. C. Longuet-Higgins, Proc. Roy. Soc. (London) A195 (1948) 188-197.

29. R. F. Langler, Chem. Educator 7 (2002) 1-6.

30. R. F. Langler, Quim. Nova 24 (2001) 416-418.

31. J. C. Paniagua and A. Moyano, J. Mol. Struct. (Theochem) 166 (1988) 113-118.

32. V. Gineityte, Int. J. Quant. Chem. 101 (2005) 274-282.

33. S. Huzinaga, The MO Method, Mir, Moscow, 1983 (in Russian).

34. V. Gineityte, Int. J. Quant. Chem. 105 (2005) 232-245.

35. V. Gineityte, Int. J. Quant. Chem. 106 (2006) 2145-2160

36. V. Gineityte, Croat. Chem. Acta 81 (2008) 487-499.

37. V. Gineityte, Croat. Chem. Acta 86 (2013) 171-185.

38. M. A. Ratner, J. Phys. Chem. 94 (1990) 4877-4883.

39. J. N. Onuchic and D. N. Beratan, J. Chem. Phys. 92 (1990) 722-733.

40. R. A. Marcus, J. Chem. Phys. 24 (1956) 966-978.

41. R. A. Marcus and N. Sutin, Biochim. Biophys. Acta 811 (1985) 265-322.

42. N. S. Hush, Progr. Inorg. Chem. 8 (1967) 391-444.

43. R. J. Cave and M. D. Newton, J. Chem. Phys. 106 (1997) 9213-9226.

44. C. Liang and M. D. Newton, J. Phys. Chem. 97 (1993) 3199-3211.

45. P. Siddarth and R. A. Marcus, J. Phys. Chem. 94 (1990) 8430-8434.

46. M. N. Paddon-Row and K. D. Jordan, in: J. F. Liebman and A. Greenberg (Eds.), Modern Models of Bonding and Delocalization, VCH Publishers, 1988, p. 115.

47. M. N. Paddon-Row, M. J. Shephard, and K. D. Jordan, J. Phys. Chem. 97 (1993) 1743-1745.

48. S. Larsson, J. Amer. Chem. Soc. 103 (1981) 4034-4040.

49. S. Larsson, J. Phys. Chem. 88 (1984) 1321-1323.

50. V. Gineityte, J. Mol. Struct. (Theochem) 343 (1995) 183-194.

51. V. Gineityte, Int. J. Quant. Chem. 68 (1998) 119-127.

52. V. Gineityte, Int. J. Quant. Chem. 81 (2001) 321-331.

53. V. Gineityte, Lithuanian J. Phys. 44 (2004) 219-236.

54. D. M. Cvetkovic, M. Doob, and H. Sachs, Spectra of Graphs. Theory and Application, VEB Deutscher Verlag der Wissenschaften, Berlin, 1980.

55. N. Trinajstic, Chemical Graph Theory, 1st ed., Parts I and II, CRC Press, Boca Raton, FL, 1983.

56. M. J. S. Dewar and R. C. Dougherty, The PMO Theory of Organic Chemistry, Plenum Press, New York, 1975.

57. J. R. Dias, J. Chem. Inf. Mod. 43 (2003) 1494-1501.

58. N. S. Ham, J. Chem. Phys. 29 (1958) 1229-1231.

59. J. Brunvoll, B. N. Cyvin, and S. J. Cyvin, Structural Chem. 3 (1992) 103-111.

60. G. G. Dyadyusha, M. L. Dekhtyar, and M. Y. Kornilov, MATCH Commun. Math. Chem. 23 (1988) 51-63.

61. E. Heilbronner, Helv. Chim. Acta 45 (1962) 1722-1725.

62. D. Cvetkovic, I. Gutman, and N. Trinajstic, J. Chem. Phys. 61 (1974) 2700-2706.

63. M. Randic, and N. Trinajstic, Croat. Chem. Acta, 67 (1994) $1-35$. 
64. Y. Kiang and E. Chen, Pure Appl. Chem. 55 (1983) 283-288.

65. E. Bodewig, Matrix Calculus, New York, Interscience Publ., 1959.

66. H. M. McConnell, J. Chem. Phys. 35 (1961) 508-515.
67. K. D. Jordan and M. N. Paddon-Row, Chem. Rev. 92 (1992) 395-410.

68. R. Hoffmann, A. Imamura, and W. H. Hehre, J. Amer. Chem Soc. 90 (1968) 1499-1509. 